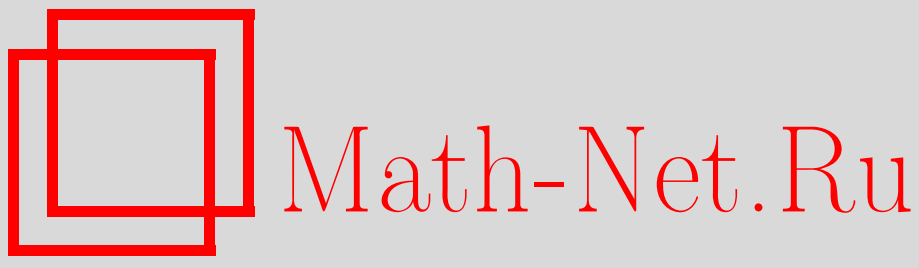

С. Н. Туманов, Асимптотические формулы для вещественных собственных значений задачи Штурма-Лиувилля с двумя точками поворота, Изв. РАН. Сер. матем., 2001, том 65, выпуск 5, 153-166

DOI: https://doi.org/10.4213/im360

Использование Общероссийского математического портала Math-Net.Ru подразумевает, что вы прочитали и согласны с пользовательским соглашением http://www . mathnet.ru/rus/agreement

Параметры загрузки:

IP : 54.224 .187 .69

26 апреля 2023 г., 17:11:40 
УДК 517.43

\author{
С. Н. Туманов
}

\title{
Асимптотические формулы для вещественных собственных значений задачи Штурма-Лиувилля с двумя точками поворота
}

\begin{abstract}
Рассмотрено асимптотическое поведение вещественного спектра при больших значениях параметра, доказано существование бесконечного числа членов асимптотики при условии аналитичности коэффициентов уравнения.

Библиография: 6 наименований.
\end{abstract}

\section{$\S 1$. Введение}

В данной работе рассматривается индефинитная задача Штурма-Лиувилля с двумя простыми точками поворота. Основной результат работы состоит в доказательстве асимптотических формул для положительных собственных значений этой задачи:

$$
u_{m} \asymp A_{1} m+A_{0}+\sum_{k=1}^{\infty} \frac{A_{-k}}{m^{k}}, \quad m \rightarrow \infty .
$$

При этом для коэффициентов $A_{n}$ имеются явные рекуррентные формулы.

В случае гладких коэффициентов и одной простой точки поворота А. А. Дородницын [2] нашел первые три члена асимптотики. Недавно А. В. Дьяченко [3] показал, что в условиях работы [2] существует бесконечно много членов асимптотики. Им же показано, что первые три члена асимптотики существуют и для двух точек поворота. Ранее в общем случае главный член был найден $\Phi$. В. Аткинсоном и А. Б. Мингарелли [1].

Итак, рассмотрим следующую краевую задачу:

$$
\begin{aligned}
& y^{\prime \prime}=\left(u^{2} f+g\right) y, \quad u>0, \\
& y\left(b_{1}\right)=y\left(b_{2}\right)=0 .
\end{aligned}
$$

Здесь $u$ - спектральный параметр, функции $f$ и $g$ аналитичны в некоторой окрестности $\Omega$ отрезка $\left[b_{1}, b_{2}\right]$ и вещественны, а для $f$ потребуем, чтобы она имела два простых нуля $a_{1}$ и $a_{2}, b_{1}<a_{1}<a_{2}<b_{2}$, и была отрицательной на интервале $\left(a_{1}, a_{2}\right)$, вне замыкания которого она предполагается положительной. Предполагаем, что эти условия, наложенные на функции $f$ и $g$, выполнены везде в настоящей работе, в том числе в формулировках теорем.

Работа выполнена при поддержке грантов РФФИ (№ 98-01-01000) и “Университеты России” (№ 992238).

$$
\text { (C) С.н. Туманов, } 2001
$$


Работа основывается на методе линий и графов Стокса, описание которого можно найти в монографии М.В. Федорюка [6]. Главным отличием построений, используемых нами, от построений, приведенных в [6], является локальность в определении асимптотических решений уравнения (1.1). Мы не требуем аналитичности рассматриваемых нами функций во всей комплексной плоскости, хотя это приводит к более сложному виду асимптотических разложений решений и матриц перехода.

В данной работе все асимптотические формулы, если это не указано явно, имеют место при $u \rightarrow+\infty$.

\section{§ 2. Граф Стокса}

Напомним ряд основных определений теории граффов Стокса.

ОПРЕДЕЛЕНИЕ 2.1. Точками поворота порядка $n$ задачи (1.1) называются нули функции $f$ кратности $n$; простыми точками поворота называются точки поворота первого порядка.

Для любой точки поворота $a$ можно ввести многозначную функцию

$$
S(a, z)=\int_{a}^{z} \sqrt{f(\zeta)} d \zeta .
$$

ОПРЕДЕЛЕНИЕ 2.2. Линией Стокса уравнения (1.1), выходящей из точки поворота $a$, называется кривая, для которой $\operatorname{Re} S(a, z)=0$.

ОПРЕДЕЛЕНИЕ 2.3. Комплексом Стокса уравнения (1.1) называется всякое максимальное связное множество, состоящее из линий Стокса. Комплекс Стокса называется простылм, если включает лишь одну точку поворота. В противном случае комплекс называется сложным.

ОПРЕДЕЛЕНИЕ 2.4. Графом Стокса уравнения (1.1) назьвается совокупность всех комплексов Стокса.

Справедливы следующие несложные утверждения, на доказательстве которых мы не будем останавливаться (ср. [6]).

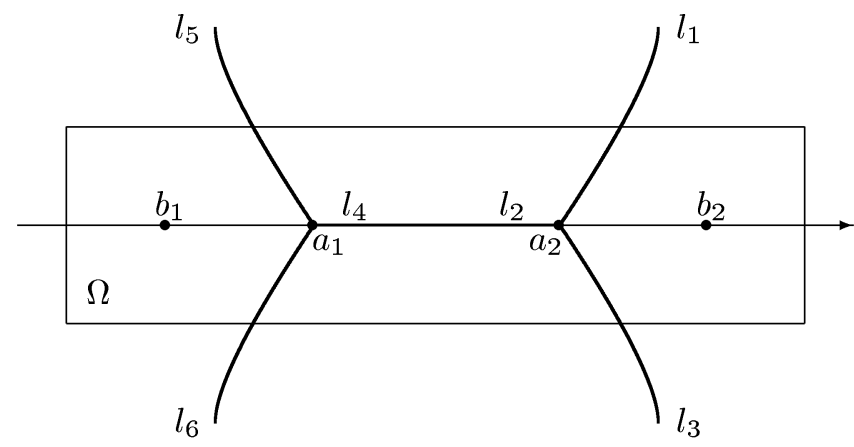

Рис. 1. Граф̆ Стокса исходного уравнения 
ЛЕмма 2.1. Из всякой простой точки поворота выходят ровно три линии Стокса под углами $\pm 2 \pi / 3$ по отношению друг к другу.

Лемма 2.2. Граф Стокса вещественной функщии $f$ симметричен относительно вещественной оси.

ЛЕмма 2.3. Пусть функиии $f$ и g удовлетворяют условиям $\S 1 ;$ тогда граф Стокса в $\Omega$ представляет собой сложный комплекс Стокса, состоящий из отрезка $\left[a_{1}, a_{2}\right]$ и четырех кривых, попарно выходящих из точек $a_{1}$ и $a_{2}$ под углами $\pm 2 \pi / 3$ по отношению к отрезку (см. рис. 1$)$.

ДоКАЗАТЕЛЬСТВО автоматически следует из предыдущих лемм и отрицательности $f$ на $\left[a_{1}, a_{2}\right]$.

Введем ряд обозначений: пусть $l_{1}, l_{2}, l_{3}$ - линии Стокса, выходящие из точки $a_{2}$ (нумерация против часовой стрелки, $\left.l_{2}=\left[a_{1}, a_{2}\right]\right) ; l_{4}, l_{5}, l_{6}$ - линии Стокса, выходящие из точки $a_{1}$ (нумерация против часовой стрелки, $l_{4}=\left[a_{1}, a_{2}\right]$ ).

Через $D_{j}$ обозначим области в $\Omega$, ограниченные линиями Стокса, содержашие внутри лишь одну линию Стокса $l_{j}$. Например, $D_{1}$ ограничена линиями $l_{5}, l_{4}=l_{2}$, $l_{3}$ и содержит $l_{1}$, а область $D_{2}$ ограничена линиями $l_{5}, l_{6}, l_{3}, l_{1}$ и содержит линию Стокса $l_{2}=\left[a_{1}, a_{2}\right]$. В дальнейшем мы будем называть эти области каноническими. Отметим, что автор понятия канонических областей, М. В. Федорюк, вводит их несколько иньм способом в $[6$, гл. $3, \S 3]$, хотя суть понятия при этом не меняется, что показывает следуюшая

ЛЕмма 2.4. Существует прямоугольная окрестность $\Omega^{\prime}$ отрезка $\left[b_{1}, b_{2}\right]$, содержащаяся в $\Omega$ и такая, что в областях $D_{j}^{\prime}=D_{j} \cap \Omega^{\prime}, j=1,2,3,4,5,6$, функиия $S\left(a_{k}, z\right), \quad k=1,2$, однолистна.

ДоКАЗАТЕльСТво. Отметим, что $S$ имеет лишь две особые точки $a_{1}$ и $a_{2}$, вне которых $S^{\prime} \neq 0$. Ввиду монотонности функции $S\left(a_{2}, z\right)$ на $\left[a_{2}, b_{2}+\varepsilon\right)$ можно гарантировать ее однолистность в некоторой прямоугольной окрестности отрезка $\left[a_{2}+\delta, b_{2}\right]$, не содержашей окрестности точки $a_{2}$ (аналогично, функция $S\left(a_{1}, z\right)$ однолистна в окрестности $\left.\left[b_{1}, a_{1}-\delta\right]\right)$. Дальше доказательство идейно повторяет доказательство леммы об однолистности в канонических областях [4]. Лемма доказана.

Без ограничения обшности мы можем считать $\Omega^{\prime}=\Omega$, так как в противном случае уменьшим исходную прямоугольную окрестность.

\section{§ 3. Канонические пары решений}

В этом параграфе мы построим пары независимых решений, разлагающихся в некоторых областях в асимптотические ряды, и найдем формулы для определения коэффициентов асимптотических разложений. Напомним ряд важных определений.

ОПРЕДЕЛЕНИЕ 3.1. Области $D_{j}$ будем называть каноническими. Тройка $\left(a_{j}, l_{j}, D_{j}\right)$ каноническая, если $a_{j} \in l_{j} \subset D_{j}$, где $a_{j}$ - точка поворота, $l_{j}$ - линия Стокса, лежащая в $D_{j}$. 
ОПРЕДЕЛЕНИЕ 3.2. Однозначную аналитическую ветвь $S_{j}(z)$ функции $S\left(a_{j}, z\right)$ в области $D_{j}$ назовем канонической, если $\operatorname{Im} S_{j}\left(l_{j}\right) \subset \mathbb{R}_{+}$.

Итак, пусть $D_{j} \subset \Omega$ - некоторая каноническая область. Выделим каноническую ветвь $S_{j}$, которая согласно лемме 2.4 будет однолистной в $D_{j}$, что, в свою очередь, позволит произвести аналитическую замену переменных (выполнить преобразование Лангера) и перейти к новому уравнению. Положим

$$
\xi=\xi(z)=S_{j}(z), \quad y=\dot{z}^{\frac{1}{2}} w .
$$

Здесь дифференцирование идет по новой независимой переменной $\xi$. В результате такой замены уравнение (1.1) примет вид

$$
w^{\prime \prime}=\left(u^{2}+\psi\right) w, \quad \psi(\xi)=g \dot{z}^{2}+\dot{z}^{\frac{1}{2}} \frac{d^{2}}{d \xi^{2}} \dot{z}^{-\frac{1}{2}} .
$$

Пусть $O_{j}=S_{j}\left(D_{j}\right)$ - область изменения новой переменной $\xi$. Очевидно, что при такой замене $\psi$ становится аналитической функцией аргумента $\xi$ в $O_{j}$.

Теорема 3.1. Уравнение (3.1) имеет следующие формальные асимптотические решения (см. $[5$, гл. $10, \S 2])$ :

$$
w_{ \pm} \sim \exp \left( \pm u \xi+\sum_{k=1}^{\infty} \frac{E_{k}(\xi)}{( \pm u)^{k}}\right) \sim e^{ \pm u \xi} \sum_{s=0}^{\infty}( \pm 1)^{s} \frac{A_{s}(\xi)}{u^{s}} .
$$

Для коэффициентов имеются следующие рекуррентные формуль:

$$
\begin{gathered}
E_{1}(\xi)=\frac{1}{2} \int \psi(\xi) d \xi, \quad E_{2}(\xi)=-\frac{1}{4} \psi(\xi), \\
E_{k+1}(\xi)=-\frac{1}{2} E_{k}^{\prime}(\xi)-\frac{1}{2} \sum_{j=1}^{k-1} \int E_{j}^{\prime}(\xi) E_{k-j}^{\prime}(\xi) d \xi, \quad k \geqslant 2 ; \\
A_{0}(\xi)=\text { const }, \quad A_{s+1}(\xi)=-\frac{1}{2} A_{s}^{\prime}(\xi)+\frac{1}{2} \int \psi(\xi) A_{s}(\xi) d \xi, \quad s \geqslant 0 .
\end{gathered}
$$

Имеет место формула связи между коэффициентами:

$$
E_{k}(\xi)=A_{k}(\xi)-\frac{1}{k} \sum_{j=1}^{k-1} j A_{k-j}(\xi) E_{j}(\xi) .
$$

ДокАЗАТЕЛЬСТво. Все формулы получаются подстановкой формальных рядов в уравнение (3.1) с последующим приравниванием коэффициентов при одинаковых степенях $u$. Теорема доказана.

Обратим внимание на то, что теорема утверждает лишш выполнимость формальных равенств для степенных рядов, но негарантирует никаких оценок на остаточные члены. Обратим также внимание на отсутствие пределов в интегралах, которые достаточно считать неопределенными.

Изучим асимптотические свойства решений. Определение и теорема, следующие ниже, приведены в [5, гл. $10, \S 3]$. 
ОПРЕДЕЛЕНИЕ 3.3. Множества $\Xi_{j}^{ \pm}\left(u, d_{j}^{ \pm}\right) \subset O_{j}$ будем называть областями справедливости асимптотических разложений, если для всех $\xi$ из $\Xi_{j}^{ \pm}\left(u, d_{j}^{ \pm}\right)$ найдутся контуры $Q_{j}^{ \pm}$, соединяюшие $d_{j}^{ \pm}$с $\xi$ и лежашие в $O_{j}$, такие, что $\operatorname{Re}(u v)$ не убывает, когда $v$ изменяется вдоль $Q_{j}^{+}$, и не возрастает, когда $v$ изменяется вдоль $Q_{j}^{-}$.

Теорема 3.2. Уравнение (3.1) имеет для всякого $и>0 u n \in \mathbb{N}$ решения $w_{n j}^{ \pm}$, аналитические в $O_{j}$, зависящие от точек $d_{j}^{ \pm}$и имеющие вид

$$
\begin{aligned}
& w_{n j}^{+}(u, \xi)=e^{u \xi} \sum_{s=0}^{n-1} \frac{A_{s}(\xi)}{u^{s}}+\varepsilon_{n j}^{+}(u, \xi), \\
& w_{n j}^{-}(u, \xi)=e^{-u \xi} \sum_{s=0}^{n-1}(-1)^{s} \frac{A_{s}(\xi)}{u^{s}}+\varepsilon_{n j}^{-}(u, \xi) .
\end{aligned}
$$

Коэффичиенты $A_{s}(\xi)$ определяются рекуррентно соотношениями

$$
A_{0}(\xi)=1, \quad A_{s+1}(\xi)=-\frac{1}{2} A_{s}^{\prime}(\xi)+\frac{1}{2} \int \psi(\xi) A_{s}(\xi) d \xi
$$

и при $\xi \in \Xi_{j}^{ \pm}\left(u, d_{j}^{ \pm}\right)$справедливь оченки на остаточные члены:

$$
\left|\varepsilon_{n j}^{ \pm}\right|,\left|\frac{\partial \varepsilon_{n j}^{ \pm}}{u \partial \xi}\right| \leqslant 2\left|e^{\mp u \xi}\right| \exp \left(\frac{2 \operatorname{Var}_{d_{j}^{ \pm}, \xi}\left(A_{1}\right)}{|u|}\right) \frac{\operatorname{Var}_{d_{j}^{ \pm}, \xi}\left(A_{n}\right)}{\left|u^{n}\right|} .
$$

Заметим, что из $[5$, гл. $10, \S 6]$ следует сушествование решений, представляющихся асимптотическими рядами.

ЛЕмма 3.1. Существует прямоугольная окрестность $\Omega^{\prime}$ отрезка $\left[b_{1}, b_{2}\right]$, содержащаяся в $\Omega$ и такая, что образы областей $D_{j}^{\prime}=D_{j} \cap \Omega^{\prime}$ функиии $S_{j}$ содержатся в областях справедливости асимптотических разложений.
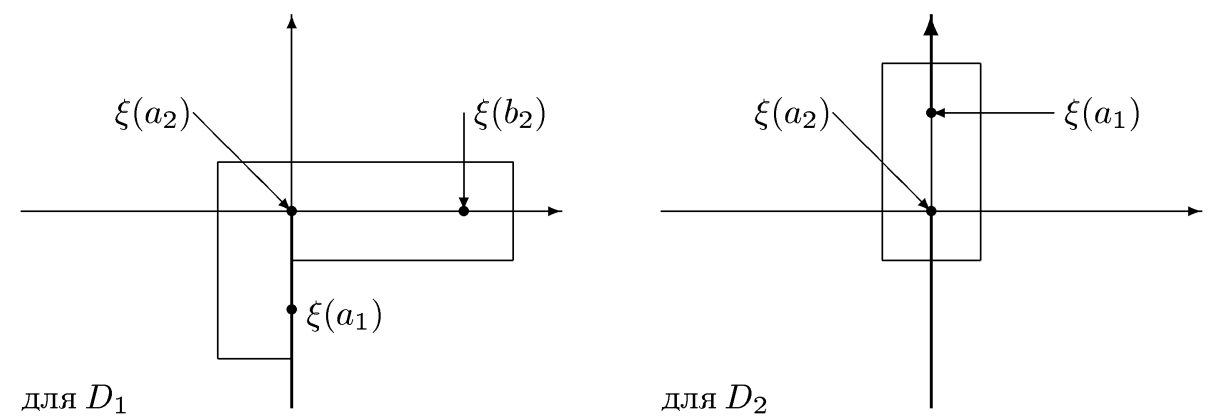

Рис. 2. Строение образов канонических областей 
ДокаЗАТЕЛЬСтво. Достаточно провести доказательство для $D_{1}$ и $D_{2}$. При малой ширине $\Omega$ в образ $D_{1}$ можно вписать угол, а в образ $D_{2}-$ прямоугольник (см. рис. 2), которые, очевидно, содержатся в областях справедливости. Заметим, что найдется прямоугольная окрестность $\Omega^{\prime}$ такая, что образы $D_{j}^{\prime}$ содержатся в соответствуюших углах и прямоугольниках. Лемма доказана.

Таким образом, не ограничивая общности, мы можем считать асимптотические формулы справедливыми всюду в образах канонических областей. Отметим, что из доказательства видно, что соответствуюшие асимптотические формулы справедливы для растушего по $u$ решения вплоть до левого берега разрезов, а для убывающего - вплоть до правого.

Из только что доказанной леммы, теоремы 3.2 и теоремы 3.1 автоматически следует

Теорема 3.3. Уравнение (3.1) имеет для всякого $и>0$ u $n \in \mathbb{N}$ решения $w_{n j}^{ \pm}$, аналитические в $O_{j}$ и представляющиеся в $O_{j}$ асимптотическими формулами

$$
w_{n j}^{ \pm}(u, \xi)=\exp \left( \pm u \xi+\sum_{s=1}^{n-1}( \pm 1)^{s} \frac{E_{s}(\xi)}{u^{s}}+O\left(\frac{1}{u^{n}}\right)\right),
$$

где коэффициенты $E_{s}(\xi)$ находятся рекуррентно согласно формулам, приведенным в теореме 3.1 .

Основываясь на этом утверждении, будем строить решения уравнения (1.1).

ТЕОРема 3.4. Пусть $D$ - каноническая область, $(a, l, D)$ - каноническая тройка, $S$ - каноническая ветвь. Тогда найдется пара решений $U$ и $V$, аналитических в $\Omega$ и представляющихся асимптотическими рядами при $z \in D$. Асимптотические формуль для $U$ также справедливы на линии Cтокса, выходящей из точки а, расположенной слева от $l$, а для $V$ - на линии Cтокса, выходящей из точки а, расположенной справа от l,

$$
\begin{aligned}
& U \asymp c f^{-1 / 4} e^{u s} \exp \left(\sum_{k=1}^{\infty} \frac{1}{u^{k}} \int^{z} \alpha_{k}(\zeta) d \zeta\right), \\
& V \asymp c f^{-1 / 4} e^{-u s} \exp \left(\sum_{k=1}^{\infty}(-1)^{k} \frac{1}{u^{k}} \int^{z} \alpha_{k}(\zeta) d \zeta\right),
\end{aligned}
$$

əде

$$
\begin{gathered}
\alpha_{0}=-\frac{1}{4} \frac{f^{\prime}}{f}, \quad \alpha_{1}=\frac{1}{2} \frac{g}{\sqrt{f}}+\frac{1}{8} \frac{f^{\prime \prime}}{\sqrt{f^{3}}}-\frac{5}{32} \frac{\left(f^{\prime}\right)^{2}}{\sqrt{f^{5}}}, \\
\alpha_{s+1}=-\frac{1}{2 \sqrt{f}}\left(\alpha_{s}^{\prime}+\sum_{j=0}^{s} \alpha_{j} \alpha_{s-j}\right), \quad s>0,
\end{gathered}
$$

а константа с выбрана так, чтобы

$$
|c|=1, \quad \lim _{l \ni z \rightarrow a} \arg \left(c f^{-1 / 4}\right)=0 .
$$


ДокАЗАТЕЛЬСтво. Рассмотрим рекуррентные формулы для $E_{s}(\xi)$. Положим $\beta_{j}=E_{j}^{\prime}$. В результате такого обозначения получим рекуррентные соотношения

$$
\beta_{1}=\frac{1}{2} \psi, \quad \beta_{2}=-\frac{1}{4} \psi^{\prime}, \quad \beta_{s+1}=-\frac{1}{2} \beta_{s}^{\prime}-\frac{1}{2} \sum_{j=1}^{s-1} \beta_{j} \beta_{s-j}
$$

В силу конформности $\xi(z)$ имеем $\xi^{\prime}(z)=f^{1 / 2}(z) \neq 0$. Положим $\alpha_{j}(z)=$ $\beta_{j}(\xi(z)) \xi^{\prime}=\beta_{j}(\xi(z)) f^{1 / 2}$. В итоге получим

$$
E_{j}(\xi)=\int^{\xi} \beta_{j}(\xi) d \xi=\int^{z} \alpha_{j}(\zeta) d \zeta
$$

После замены, обратной к преобразованию Лангера, и умножения на нормировочный множитель $c$ получим пару независимых (при больших $u$ ) решений $U$ и $V$. Соотношения на $\alpha_{j}$ следуют из соотношений на $\beta_{j}$ после выражения $\psi$ через исходные функции:

$$
\psi(\xi)=\frac{g}{f}-\frac{1}{f^{3 / 4}} \frac{d^{2}}{d z^{2}} \frac{1}{f^{1 / 4}}
$$

и введения дополнительно $\alpha_{0}=-\frac{1}{4} \frac{f^{\prime}}{f}$. Теорема доказана.

ЛЕмма 3.2. При изменении ветви $\sqrt{f}$ знак $\alpha_{j}$ меняется на $(-1)^{j}$.

ДокаЗАТЕльство. Для $j=0$ и $j=1$ утверждение очевидно. Далее по индукции имеем

$$
\alpha_{s+1}^{-}=-\frac{1}{-2 \sqrt{f}}\left((-1)^{s}\left(\alpha_{s}^{+}\right)^{\prime}+\sum_{j=1}^{s}(-1)^{j}(-1)^{s-j} \alpha_{j}^{+} \alpha_{s-j}^{+}\right)=(-1)^{s+1} \alpha_{s+1}^{+} \text {, }
$$

где выбор + или - в $\alpha_{s}^{ \pm}$зависит от выбора ветви $\sqrt{f}$. Лемма доказана.

СЛЕДСТВИЕ 3.1. Коэффициенты $\alpha_{2 k}$ над $\Omega$ распадаются на однозначнье голоморфные ветви.

Будем считать $\alpha_{2 k}$ однозначной функцией, а $\alpha_{2 k+1}$ - двузначной. Введем следуюшее обозначение: $\alpha_{k}^{(j)}$ - ветвь в канонической области $D_{j}$, в формулах определения которой фигурирует именно та ветвь $\sqrt{f}$, которая участвует в определении $S_{j}$.

Для каждой канонической тройки $\left(a_{j}, l_{j}, D_{j}\right)$ введем точки $z_{j}^{-}$и $z_{j}^{+}$, лежащие на соседних с $l_{j}$ линиях Стокса, выходящих из $a_{j}\left(z_{j}^{-}-\right.$слева от $l_{j}$, а $z_{j}^{+}-$справа), причем так, чтобы для двух линий Стокса $l_{j}, l_{j+1}\left(l_{j+1}\right.$ слева от $\left.l_{j}\right) z_{j+1}^{-}=z_{j}^{+}($см. рис. 3).

Пусть $\gamma_{j}^{ \pm}(z)$ - контур, соединяющий $z_{j}^{\mp}$ с $z$, лежащий в канонической области $D_{j}$.

ОПРЕДЕЛЕНИЕ 3.4. Пара решений дифференциального уравнения (1.1) $U_{j}$ и $V_{j}$, определенных в $\Omega$, называется канонической парой (или парой канонических 


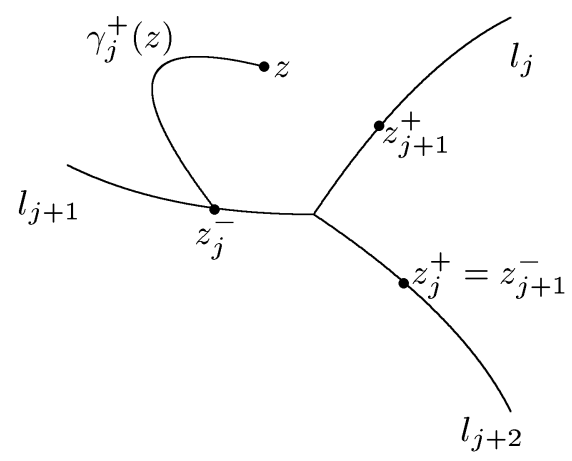

Рис. 3. Пути для канонических решений

решений) в канонической области $D_{j}$, если для всех $z \in D_{j}$ выполнены асимптотические соотношения

$$
\begin{aligned}
& U_{j}(u, z) \asymp c_{j} f^{-1 / 4} e^{u S_{j}(z)} \exp \left(\sum_{s=1}^{\infty} u^{-s} \int_{\gamma_{j}^{+}(z)} \alpha_{s}^{(j)}(\zeta) d \zeta\right), \\
& V_{j}(u, z) \asymp c_{j} f^{-1 / 4} e^{-u S_{j}(z)} \exp \left(\sum_{s=1}^{\infty}(-u)^{-s} \int_{\gamma_{j}^{-}(z)} \alpha_{s}^{(j)}(\zeta) d \zeta\right),
\end{aligned}
$$

где $c_{j}$ и $\alpha_{s}^{(j)}$ выбраны, как и в теореме 3.4 , а контуры интегрирования определены в предыдушем абзаце.

Канонические решения являются аналитическими всюду в $\Omega$, но в асимптотические ряды раскладываются только в соответствуюших канонических областях. Нашей дальнейшей задачей будет изучение поведения этих пар в других областях.

\section{§ 4. Формулы перехода}

ОПРЕДЕЛЕНИЕ 4.1. Пусть заданы пары канонических решений и канонические области: $\left(U_{j}, V_{j}\right), D_{j}$ и $\left(U_{k}, V_{k}\right), D_{k}$. Так как произвольное решение $w$ уравнения (1.1) разлагается в линейную комбинацию фундаментальных решений, то

$$
w=\alpha_{j} U_{j}+\beta_{j} V_{j}=\alpha_{k} U_{k}+\beta_{k} V_{k},
$$

где $\alpha_{l}$ и $\beta_{l}$ зависят лишш от параметра $u$. Имеем

$$
\left(\begin{array}{c}
\alpha_{k} \\
\beta_{k}
\end{array}\right)=\Omega_{j k}(u)\left(\begin{array}{c}
\alpha_{j} \\
\beta_{j}
\end{array}\right) .
$$

Следуя [6], будем называть матрицы $\Omega_{j k}(u)$ матрицами перехода от канонической тройки $\left(a_{j}, l_{j}, D_{j}\right)$ к тройке $\left(a_{k}, l_{k}, D_{k}\right)$.

Нетрудно понять, что справедливо следуюшее соотношение:

$$
\left(\begin{array}{c}
U_{j}(u, z) \\
V_{j}(u, z)
\end{array}\right)=\Omega_{j k}^{T}(u)\left(\begin{array}{c}
U_{k}(u, z) \\
V_{k}(u, z)
\end{array}\right) .
$$

Мы построим матрицы перехода между областями двух типов. Остальные будут выражаться как композиция основных. 
ТЕОРема 4.1. Пусть а - простая точка поворота, $l_{1}, l_{2}, l_{3}$ - линии Стокса с началом в точке а. Пусть $l_{j+1}$ располагается слева от $l_{j}(j \bmod 3)$, a канонические области выбраны так, чтобы часть $D_{j}$, лежсаия слева от $l_{j}$, совпадала с частью $D_{j+1}$, лежсащей справа от $l_{j+1}$. Обозначим $\Omega_{j, j+1}$ матрицу перехода от $\left(a, l_{j}, D_{j}\right) \kappa\left(a, l_{j+1}, D_{j+1}\right)$. Тогда

$$
\Omega_{j, j+1}=e^{-i \frac{\pi}{6}}\left(\begin{array}{cc}
0 & \alpha_{j, j+1}^{-1} \\
\beta_{j, j+1} & i \alpha_{j+1, j+2} / \beta_{j+2, j}
\end{array}\right),
$$

$\alpha_{12} \alpha_{23} \alpha_{31}=\beta_{12} \beta_{23} \beta_{31}=1+o(1)$ при $u \rightarrow+\infty$. Для элементов матрици перехода имеют место асимптотические формуль

$$
\begin{aligned}
& \alpha_{j, j+1} \asymp \exp \left(\sum_{k=1}^{\infty}(-u)^{-k} \int_{\gamma_{z_{j}^{+} z_{j+1}^{-}}} \alpha_{k}^{(j)} d t\right), \\
& \beta_{j, j+1} \asymp \exp \left(\sum_{k=1}^{\infty} u^{-k} \int_{\gamma_{z_{j} z_{j+1}^{+}}} \alpha_{k}^{(j)} d t\right),
\end{aligned}
$$

где контуры интегрирования $\gamma_{z_{j}^{+} z_{j+1}^{-}} u \gamma_{z_{j}^{-}} z_{j+1}^{+}$соединяют точки, обозначенные в индексах, причем первый контур огибает точку поворота по часовой стрелке, ветвь $\alpha_{k}^{(j)}$ продолжсается из области $D_{j}$ в окрестность точки поворота а без разреза $l_{j+2}$, а контур $\gamma_{z_{j}^{-}} z_{j+1}^{+}$полностью расположен $в$ $D_{j} \cap D_{j+1}$ и тохе ориентирован по часовой стрелке.

ДокАЗАТЕЛЬСтво. Введенные канонические пары разлагаются в асимптотические ряды (см. определение 3.4$)$. Пусть $D_{j, j+1}=D_{j} \cap D_{j+1}$ - область, в которой справедливы разложения для обеих канонических пар.

В предположении совпадения ветвей $f^{-1 / 4}$ получим $c_{j}=e^{-i \frac{\pi}{6}} \mathrm{c}_{j+1}$. Положим

$$
\begin{gathered}
Q_{j, j+1}=\left(\begin{array}{cc}
q_{11}^{(j)} & q_{12}^{(j)} \\
q_{21}^{(j)} & q_{22}^{(j)}
\end{array}\right)=e^{i \frac{\pi}{6}} \Omega_{j, j+1}^{T}, \\
\eta_{k}^{+}=\frac{U_{k}}{c_{k} f^{-1 / 4} e^{u S_{k}}}, \quad \eta_{k}^{-}=\frac{V_{k}}{c_{k} f^{-1 / 4} e^{-u S_{k}}}, \quad \eta_{k}^{ \pm} \sim 1 .
\end{gathered}
$$

Получим следуюшее соотношение:

$$
\left(\begin{array}{c}
e^{u S_{j}} \eta_{j}^{+} \\
e^{-u S_{j}} \eta_{j}^{-}
\end{array}\right)=\left(\begin{array}{cc}
q_{11}^{(j)} & q_{12}^{(j)} \\
q_{21}^{(j)} & q_{22}^{(j)}
\end{array}\right)\left(\begin{array}{c}
e^{u S_{j+1}} \eta_{j+1}^{+} \\
e^{-u S_{j+1}} \eta_{j+1}^{-}
\end{array}\right)
$$

Отметим, что всюду в $D_{j, j+1}$ выполнено равенство $S_{j+1}=-S_{j}$. Рассмотрим первую строчку данного соотношения и, учитывая это замечание и домножив обе стороны равенства на $e^{u S_{j+1}}$, получим

$$
\eta_{j}^{+}=q_{11}^{(j)} e^{2 u S_{j+1}} \eta_{j+1}^{+}+q_{12}^{(j)} \eta_{j+1}^{-}
$$


Заметим, что из этого соотношения немедленно следует ограниченность $q_{12}^{(j)}$. Если бы это было не так, то, взяв $z \in D_{j, j+1}$, мы бы получили

$$
\lim _{u \rightarrow+\infty} \frac{q_{11}^{(j)}}{q_{12}^{(j)}}=0
$$

что, очевидно, не выполнено при $z \in l_{j+1}$. (Асимптотическое соотношение для $U_{j}$ выполнено вплоть до $l_{j+1}$.)

Из этого вытекает, что существует $\beta>0$ такое, что $\left|q_{11}^{(j)}\right| \leqslant C e^{-\beta^{2} u}$, и, более того, всюду в $D_{j, j+1}$ сушествует $\beta>0$ такое, что $\left|q_{11}^{(j)} e^{2 u S_{j+1}}\right| \leqslant C e^{-\beta^{2} u}$. Это неравенство говорит о том, что вклад первого слагаемого равен асимптотическому нулю и $\eta_{j}^{+} \asymp q_{12}^{(j)} \eta_{j+1}^{-}$. Отсюда немедленно следует разложение

$$
q_{12}^{(j)} \asymp \exp \left(\sum_{k=1}^{\infty} u^{-k} \int_{\gamma_{z_{j}^{-} z_{j+1}^{+}}} \alpha_{k}^{(j)} d t\right) .
$$

Вместо $U_{j}$ мы рассмотрим новое решение $W_{j}=e^{-i \pi / 6} q_{12}^{(j)} V_{j+1}$, асимптотическое разложение которого совпадает с $U_{j}$, и, следовательно, $W_{j}$ является каноническим. В итоге матрищу $Q_{j, j+1}$ мы представим в виде

$$
Q_{j, j+1}=\left(\begin{array}{cc}
0 & q_{j}(u) \\
a_{j}(u) & b_{j}(u)
\end{array}\right)
$$

Будем строить матрицы перехода так, чтобы было выполнено естественное соотношение

$$
\Omega_{j, j+1}^{T} \Omega_{j+1, j+2}^{T} \Omega_{j+2, j}^{T}=I,
$$

из которого немедленно следуют эквивалентные ему равенства

$$
\forall j(\bmod 3)\left\{\begin{array}{l}
a_{j} q_{j+1} b_{j+2}=i, \\
b_{j} b_{j+1} b_{j+2}=-i
\end{array}\right.
$$

а вводя новые обозначения $a_{j}=\alpha_{j, j+1}^{-1}$ и $q_{j}=\beta_{j, j+1}$, получим вид матрицы перехода такой же, как и в условии теоремы. Из приведенной выше системы и введенных обозначений немедленно следует мультипликативное соотношение на $\alpha_{k, k+1}$ и $\beta_{k, k+1}$.

Отметим, что данное соотношение дает нам $\alpha_{j, j+1} \sim 1$, из чего следует асимптотическое равенство

$$
e^{u S_{j+1}} \eta_{j}^{+} \asymp \frac{1}{\alpha_{j, j+1}} e^{u S_{j+1}} \eta_{j+1}^{+},
$$

которое и дает представление для $\alpha_{j, j+1}$. Теорема доказана. 
Теорема 4.2. Пусть $\Omega_{j, j+1}$ - матрица перехода от тройки $\left(a_{j}, l=l_{j}\right.$, $\left.D=D_{j}\right) \kappa$ тройке $\left(a_{j+1}, l=l_{j+1}, D=D_{j+1}\right)$, т.е. $l$ соединяет точки поворота $a_{j}$ и $a_{j+1}$. Тогда $\Omega_{j, j+1}$ представляется в виде

$$
\Omega_{j, j+1}=e^{i \psi}\left(\begin{array}{cc}
0 & \alpha_{j, j+1} e^{-i u a} \\
\beta_{j, j+1} e^{i a u} & 0
\end{array}\right)
$$

$2 \partial e$

$$
\begin{gathered}
a=\left|\int_{a_{j}}^{a_{j+1}} \sqrt{f(\zeta)} d \zeta\right|, \\
\alpha_{j, j+1} \asymp \exp \left(\sum_{k=1}^{\infty}(-u)^{-k} \int_{\gamma_{z_{j} z_{j+1}^{-}}} \alpha_{k}^{(j)} d t\right), \\
\beta_{j, j+1} \asymp \exp \left(\sum_{k=1}^{\infty} u^{-k} \int_{\gamma_{z_{j} z_{j+1}^{+}}} \alpha_{k}^{(j)} d t\right),
\end{gathered}
$$

где через $\gamma_{z_{1} z_{2}}$ обозначается контур, соединяющий $z_{1} u z_{2}$, обозначенные в индексах, и челиком лежсащий в области D.

ДокАЗАтЕльство. Во-первых, обратим внимание на равенство $S_{j+1}(z)=i a-$ $S_{j}(z)$, которое немедленно следует из определения 3.2 канонической ветви. Вовторых, области справедливости асимптотических формул для пар $U_{k}$ и $V_{k}$ при $k=j, j+1$ совпадают, что позволит нам искать решения в виде

$$
U_{j}=p(u) V_{j+1}, \quad V_{j}=q(u) U_{j+1}
$$

Пусть $c_{j}=e^{i \psi} c_{j+1}$. Тогда из приведенного вьше соотношения вьведем асимптотики для коэффициентов аналогично процедуре, использованной в теореме 4.1. Теорема доказана.

\section{$\S 5$. Асимптотика собственных значений}

Теперь мы вернемся к тем обозначениям, которые были введены в $\S 2$. Для определения собственных значений нашей задачи необходимо и достаточно решить следующее уравнение:

$$
\Delta=\left|\begin{array}{ll}
U_{1}\left(u, b_{1}\right) & V_{1}\left(u, b_{1}\right) \\
U_{1}\left(u, b_{2}\right) & V_{1}\left(u, b_{2}\right)
\end{array}\right|=0
$$

где $\Delta$ - характеристический определитель системы (1.1). Так как фундаментальные решения $U_{1}$ и $V_{1}$ нам известны своими асимптотиками только в области $D_{1}$, содержашей $b_{2}$, то нам придется выражать эти решения через каноническую пару $U_{5}, V_{5}$, для которой известно асимптотическое поведение в области $D_{5}$, содержащей точку $b_{1}$, т.е. найти матрицу перехода $\Omega_{15}$. Очевидно, что

$$
\Omega_{15}^{T}=\Omega_{12}^{T} \Omega_{24}^{T} \Omega_{45}^{T}
$$


где вид матрищ $\Omega_{12}$ и $\Omega_{45}$ следует из теоремы 4.1 , а вид матрищы $\Omega_{24}$ следует из теоремы 4.2. Таким образом,

$$
\Omega_{15}^{T}=e^{i \gamma}\left(\begin{array}{cc}
0 & \beta_{12} \\
\alpha_{12}^{-1} & i \alpha_{23} / \beta_{31}
\end{array}\right)\left(\begin{array}{cc}
0 & \beta_{24} e^{i u a} \\
\alpha_{24} e^{-i u a} & 0
\end{array}\right)\left(\begin{array}{cc}
0 & \beta_{45} \\
\alpha_{45}^{-1} & i \alpha_{56} / \beta_{64}
\end{array}\right),
$$

что после вычислений дает

$\Omega_{15}^{T}=e^{i \gamma}\left(\begin{array}{cc}0 & \alpha_{24} \beta_{12} \beta_{45} e^{-i u a} \\ \frac{\beta_{24}}{\alpha_{12} \alpha_{45}} e^{i u a} & i \frac{\alpha_{56} \beta_{24}}{\alpha_{12} \beta_{64}} e^{i u a}+i \frac{\alpha_{23} \alpha_{24} \beta_{45}}{\beta_{31}} e^{-i u a}\end{array}\right), \quad\left(\begin{array}{c}U_{1} \\ V_{1}\end{array}\right)=\Omega_{15}^{T}\left(\begin{array}{c}U_{5} \\ V_{5}\end{array}\right)$.

Теперь можно расписать характеристический определитель и получить уравнение

$$
\omega_{11} U_{5}\left(b_{1}\right) V_{1}\left(b_{2}\right)+\omega_{21} V_{5}\left(b_{1}\right) V_{1}\left(b_{2}\right)=\omega_{12} U_{5}\left(b_{1}\right) U_{1}\left(b_{2}\right)+\omega_{22} V_{5}\left(b_{1}\right) U_{1}\left(b_{2}\right) .
$$

Очевидно, что $\operatorname{Re} S_{1}\left(b_{2}\right)>0$ и $\operatorname{Re} S_{5}\left(b_{1}\right)<0$, т.е. $\operatorname{Re}\left(-S_{4}\left(b_{1}\right)+S_{1}\left(b_{2}\right)\right)-$ наибольшее из чисел $\operatorname{Re}\left( \pm S_{4}\left(b_{1}\right) \pm S_{1}\left(b_{2}\right)\right)$, где первый и второй знаки выбираются произвольно. Вьвод: в (5.1) главным является слагаемое $\omega_{22} V_{5}\left(b_{1}\right) U_{1}\left(b_{2}\right)$. Таким образом, при больших значениях $u$ это уравнение эквивалентно более простому: $\omega_{22}=0$, или

$$
\frac{\alpha_{56} \beta_{24}}{\alpha_{12} \beta_{64}} e^{2 i u a}+\frac{\alpha_{23} \alpha_{24} \beta_{45}}{\beta_{31}}=0,
$$

что, в свою очередь, эквивалентно

$$
\frac{\beta_{24}}{\alpha_{24}} \frac{\alpha_{56}}{\beta_{64} \beta_{45}} \frac{\alpha_{31}}{\beta_{12} \beta_{23}} e^{2 i u a}=-1 .
$$

Удобно ввести обозначения: $\alpha_{k}=\alpha_{k}^{(1)}, \gamma_{\left[a_{1}, a_{2}\right]}$ - замкнутьй контур в $\Omega$, обходящий отрезок $\left[a_{1}, a_{2}\right]$ в направлении против часовой стрелки.

ЛЕмма 5.1. При $и \rightarrow+\infty$ справедливо следующее асимптотическое уравнение на положительные собственные значения оператора Штурма-Лиувилля с двумя точками поворота:

$$
e^{2 i u a} \asymp-\exp \left(\sum_{k=1}^{\infty} \frac{1}{u^{k}} \oint_{\gamma_{\left[a_{1}, a_{2}\right]}} \alpha_{k} d t\right) .
$$

ДоКАЗАТЕЛЬСТво следует из уравнения (5.2) после подстановки асимптотических формул для всех $\alpha_{i j}$ и $\beta_{i j}$, введенных в $\S 4$.

Лемма 5.2. Пусть $\rho_{m} \rightarrow+\infty$ при $m \rightarrow \infty$. Тогда из асимптотического уравнения

$$
e^{2 i \rho_{m} a}+1 \asymp \sum_{k=1}^{\infty} \frac{p_{k}}{\rho_{m}^{k}}
$$

следует представление для $\rho_{m}$ в виде асимптотического ряда:

$$
\rho_{m} \asymp A_{1} m+A_{0}+\sum_{k=1}^{\infty} \frac{A_{-k}}{m^{k}},
$$

где коэффичиенты $A_{s}$ определяются рекуррентно через $p_{k}$. 
ДокАЗАТЕЛЬСТво. Фиксируем некоторое натуральное $k$. Тогда асимптотическое равенство можно переписать в виде

$$
e^{2 i \rho_{m} a-2 \pi i(m-s)-\pi i}=1+\frac{-p_{1}}{\rho_{m}}+\cdots+\frac{-p_{k}}{\rho_{m}^{k}}+o\left(\frac{1}{\rho_{m}^{k}}\right),
$$

где $s \in \mathbb{Z} ;$ логарифмируя равенство, получаем следующую формулу:

или

$$
2 i \rho_{m} a-2 \pi i(m-s)-\pi i=\frac{q_{1}}{\rho_{m}}+\cdots+\frac{q_{k}}{\rho_{m}^{k}}+o\left(\frac{1}{\rho_{m}^{k}}\right)
$$

$$
\rho_{m}=b_{0}(m-s)+b_{1}+\frac{r_{1}}{\rho_{m}}+\cdots+\frac{r_{k}}{\rho_{m}^{k}}+o\left(\frac{1}{\rho_{m}^{k}}\right) .
$$

Выразим $m$ из правой части равенства и возведем его в отрицательные целые степени, после чего получим следующую систему равенств:

$$
\begin{aligned}
& \frac{1}{m}=\frac{s_{11}}{\rho_{m}}+\frac{s_{12}}{\rho_{m}^{2}}+\cdots+\frac{s_{1 k}}{\rho_{m}^{k}}+o\left(\frac{1}{\rho_{m}^{k}}\right), \\
& \frac{1}{m^{2}}=\quad \frac{s_{22}}{\rho_{m}^{2}}+\cdots+\frac{s_{2 k}}{\rho_{m}^{k}}+o\left(\frac{1}{\rho_{m}^{k}}\right), \\
& \frac{1}{m^{k}}= \\
& \frac{s_{k k}}{\rho_{m}^{k}}+o\left(\frac{1}{\rho_{m}^{k}}\right) .
\end{aligned}
$$

Данная система разрешима относительно обратных степеней $\rho_{m}$, в частности, имеем

$$
\frac{1}{\rho_{m}}=\frac{d_{1}}{m}+\cdots+\frac{d_{k}}{m^{k}}+o\left(\frac{1}{\rho_{m}^{k}}\right)
$$

но $o\left(\frac{1}{\rho_{m}^{k}}\right)=o\left(\frac{1}{m^{k}}\right)$, а обрашение получившегося равенства уже не вызывает сомнения. Лемма докзана.

ТЕорема 5.1. Для положительных собственных значений оператора Штурма-Лиувилля с двумя простыми точками поворота справедливо асимптотическое представление

$$
u_{m} \asymp A_{1} m+A_{0}+\sum_{k=1}^{\infty} \frac{A_{-k}}{m^{k}}, \quad m \rightarrow \infty,
$$

в частности, с точностью до $O\left(\mathrm{~m}^{-3}\right)$,

$$
\begin{aligned}
u_{m}= & \frac{1}{\int_{a_{1}}^{a_{2}} \sqrt{-f} d t}\left(\pi m+\frac{\pi}{2}\right)+\frac{1}{m} \frac{1}{2 \pi i} \oint_{\gamma_{\left[a_{1}, a_{2}\right]}}\left(\frac{1}{2} \frac{g}{f^{1 / 2}}+\frac{1}{8} \frac{f^{\prime \prime}}{f^{3 / 2}}-\frac{5}{32} \frac{\left(f^{\prime}\right)^{2}}{f^{5 / 2}}\right) d t \\
& +\frac{1}{m^{2}} \frac{1}{2 \pi i}\left(\frac{1}{\pi} \int_{a_{1}}^{a_{2}} \sqrt{-f} d t \oint_{\gamma_{\left[a_{1}, a_{2}\right]}} \alpha_{2} d t-\frac{1}{2} \oint_{\gamma_{\left[a_{1}, a_{2}\right]}} \alpha_{1} d t\right)+O\left(\frac{1}{m^{3}}\right),
\end{aligned}
$$

где ветвь $f^{1 / 2}$ положительна при $z>a_{2}$, значения $\sqrt{-f}$ положительны на всем интервале $\left(a_{1}, a_{2}\right)$, замкнутый контур $\gamma_{\left[a_{1}, a_{2}\right]}$ лежит в $\Omega$ и обходит $\left[a_{1}, a_{2}\right]$ против часовой стрелки; $\alpha_{j}$ определены формулами (3.2), ветвь $\sqrt{f(z)}$ в определении $\alpha_{j}$ положительна при $z>a_{2}$.

Для определения остальных коэффициентов достаточно выписать асимптотическое разложение $u_{m}$ с неопределенными коэффициентами, подставить в (5.3) и разрешить получившееся уравнение, приравнивая слагаемые, стоящие при равных степенях $m$. 
ДокАЗАТЕЛЬСТво. Первое утверждение теоремы немедленно следует из предыдущей леммы. Для определения коэффициентов разложения $u_{m}$ будем выписывать его с неопределенными коэффициентами и подставлять в (5.3). Например,

$$
e^{2 i u_{m} a}=-1+O\left(u^{-1}\right)
$$

что дает

$$
u_{m}=\frac{1}{a}\left(\pi\left(m-m_{0}\right)+\frac{\pi}{2}\right)+O\left(m^{-1}\right),
$$

где согласно [1] $m_{0}=0$. Для вычисления коэффициента $A_{-1}$ подставим выражение

$$
u_{m}=\frac{1}{a}\left(\pi m+\frac{\pi}{2}\right)+\frac{A_{-1}}{m}+O\left(m^{-2}\right)
$$

в (5.3) и приравняем коэффициенты при $m^{-1}$. Тогда получим

$$
A_{-1}=\frac{1}{2 \pi i} \oint_{\gamma_{\left[a_{1}, a_{2}\right]}} \alpha_{1} d t .
$$

Зная выражения для коэффициентов $A_{0}, A_{-1}$, находим $A_{-2}$, а затем и последующие коэффициенты. Теорема доказана.

Автор благодарит проф. А. А. Шкаликова за постановку задачи, ценные замечания и советы.

\section{Список литературы}

1. Atkinson F. V., Mingarelli A.B. Asymptotic of the number of zeros and of eigenvalues of general weight Sturm-Liouville problems // J. reine ang. Math. 1987. V. 375. P. 380-393.

2. Дородницын А. А. Асимптотические законы распределения собственных значений для некоторых особых видов дифференциальных уравнений второго порядка // УМН. 1952. T. 7. №6. C. 3-96.

3. Дьяченко А. В. Асимптотика собственных значений индефинитной задачи Штурма-Лиувилля // Матем. заметки. 2000. Т. 68. №1. С. 139-143.

4. Евграфов М. А., Федорюк M.В. Асимптотика решений уравнения $w^{\prime \prime}-p(z, \lambda) w=0$ при $\lambda \rightarrow \infty$ в комплексной плоскости // УМН. 1966. Т. 21. № 1. С. 3-50.

5. Олвер $\Phi$. Асимптотика и специальные функции. М.: Наука, 1990.

6. Федорюк М. В. Асимптотические методы для линейных обыкновенных диффференциальных уравнений. М.: Наука, 1983. 